Journal of Organometallic Chemistry, 431 (1992) 143-150

Elsevier Sequoia S.A., Lausanne

JOM 22554

\title{
Synthese und strukturelle Charakterisierung von bcpm-Metallcarbonyl-Dimeren (bcpm = Biscyclopentadienylmethan) mit Metallen der 6. Nebengruppe
}

\author{
Henri Brunner, Roland Graßl, Joachim Wachter \\ Institut für Anorganische Chemie der Universität Regensburg, Universitätsstraße 31, \\ W-8400 Regensburg (Deutschland)
}

\section{Bernd Nuber und Manfred L. Ziegler}

Anorganisch-chemisches Institut der Universität Heidelberg, Im Neuenheimer Feld 270, W-6900 Heidelberg (Deutschland)

(Eingegangen den 29. November 1991)

\begin{abstract}
The reaction of methylendicyclopentadiene $\left(b c p m H_{2}\right)$ with $\left(\mathrm{CH}_{3} \mathrm{CN}\right)_{3} \mathrm{M}(\mathrm{CO})_{3}(\mathrm{M}=\mathrm{Cr}, \mathrm{Mo}$, W) gives the complexes bcpmM ${ }_{2}(\mathrm{CO})_{6}(1-3)$ in good yields $(47-78 \%)$. The synthesis of bcpmMo ${ }_{2}\left(\mathrm{CO}_{6} \mathrm{Cl}_{2}\right.$ (4) starts from bcpmLi 2 and $\mathrm{Mo}(\mathrm{CO})_{6}$. In a one-pot procedure, acidification with $\mathrm{CH}_{3} \mathrm{CO}_{2} \mathrm{H}$ and reaction with $\mathrm{CCl}_{4}$ give 4 in $43 \%$ yield. Low temperature ${ }^{1} \mathrm{H}$ NMR investigations of 1 and 2 reveal a dynamic process analogous to that already observed for the silylbridged homologues. The $\mathrm{X}$-ray analysis of 1 proves the chiral structure of the molecule, which is the consequence of the distortion of the bcpm-ligand. The $\mathrm{Cr}-\mathrm{Cr}$ distance is $3.211(2) \AA$.
\end{abstract}

\section{Zusammenfassung}

Die Reaktion von Methylendicyclopentadien $\left(\mathrm{bcpmH}_{2}\right)$ mit $\left(\mathrm{CH}_{3} \mathrm{CN}\right)_{3} \mathrm{M}(\mathrm{CO})_{3}(\mathrm{M}=\mathrm{Cr}$, $\mathrm{Mo}, \mathrm{W})$ führt zu den Komplexen bcpmM ${ }_{2}(\mathrm{CO})_{6}(1-3)$ in guten Ausbeuten (47-78\%). Die Synthese von bepmMo ${ }_{2}(\mathrm{CO})_{6} \mathrm{Cl}_{2}(4)$ geht von bcpmLi ${ }_{2}$ und $\mathrm{Mo}(\mathrm{CO})_{6}$ aus und liefert in einem Eintopfverfahren nach Ansäuern mit $\mathrm{CH}_{3} \mathrm{CO}_{2} \mathrm{H}$ und Umsetzung mit $\mathrm{CCl}_{4} 4$ in $43 \%$ Ausbeute. Tieftemperatur- ${ }^{-1} \mathrm{H}-\mathrm{NMR}$ spektroskopische Untersuchungen an 1 und 2 decken einen dynamischen ProzeB auf, analog zu den bereits früher untersuchten silylverbrückten Homologen. Die Röntgenstrukturanalyse von 1 beweist den chiralen Aufbau des Moleküls, der durch die Verwindung des bcpm-Liganden zustande kommt. Der Cr-Cr-Abstand beträgt 3.211(2) A.

\section{Einleitung}

Die Chemie von Cyclopentadienyl-Übergangsmetallcarbonyl-Dimeren, in denen die $\pi$-Liganden durch Methylen- oder Dimethylsilylbrücken verknüpft sind, besitzt

Correspondence to: Dr. J. Wachter, Institut für Anorganische Chemie der Universität Regensburg, Universitätsstraße 31, W-8400 Regensburg, Deutschland. 
gegenüber ihren unverbrückten Grundkörpern zusätzliche interessante Aspekte. Hierfür sind vor allem kooperative Effekte zwischen den Metallnachbarn sowie eine stereochemische Kontrolle durch den Brückenliganden verantwortlich. Bereits synthetisiert worden sind die Methylen-verbrückten Analoga von $\mathrm{Cp}_{2} \mathrm{Fe}_{2}(\mathrm{CO})_{4}$ [1], $\mathrm{Cp}_{2} \mathrm{Ru}_{2}(\mathrm{CO})_{4}$ [2], $\mathrm{Cp}_{2} \mathrm{Co}_{2}(\mathrm{CO})_{2}$ [3], $\mathrm{Cp}_{2} \mathrm{Rh}_{2}(\mathrm{CO})_{3}$ [4], $\mathrm{Cp}_{2} \mathrm{Ir}_{2}(\mathrm{CO})_{3}$ [5] (Cp = $\left.\mathrm{C}_{5} \mathrm{H}_{5}\right)$ und $\mathrm{Cp}_{2}{ }_{2} \mathrm{Rh}_{2}(\mathrm{CO})_{2}[6]\left(\mathrm{Cp}^{\star}=\mathrm{C}_{5} \mathrm{Me}_{5}\right)$. Von den Dimeren der 6. Nebengruppe sind bisher lediglich die Dimethylsilylkomplexe $\mathrm{Me}_{2} \mathrm{Si}\left[\left(\mathrm{C}_{5} \mathrm{H}_{4}\right) \mathrm{M}(\mathrm{CO})_{3}\right]_{2}$ $(\mathrm{M}=\mathrm{Cr}, \mathrm{Mo}, \mathrm{W})$ beschrieben worden [7]. Im folgenden wird erstmals über eine ergiebige Synthese der $\mathrm{CH}_{2}$-verbrückten Derivate sowie deren spektroskopische und strukturelle Eigenschaften berichtet. Die hierbei gewonnenen Erkenntnisse werden auf die Darstellung der hierzu nahe verwandten Verbindung bcpmMo ${ }_{2}$ $(\mathrm{CO})_{6} \mathrm{Cl}_{2}$ angewendet.

\section{Präparative und spektroskopische Ergebnisse}

bcpmM $_{2}(\mathrm{CO})_{6}{ }^{- \text {Komplexe }}(\mathrm{M}=\mathrm{Cr}, \mathrm{Mo}, \mathrm{W})$

Die Synthesestrategie beruht wegen der Thermolabilität von Methylendicyclopentadien auf der Umsetzung des Liganden mit den Trisacetonitril-Komplexen $\left(\mathrm{CH}_{3} \mathrm{CN}\right)_{3} \mathrm{M}(\mathrm{CO})_{3}(\mathrm{M}=\mathrm{Cr}$, Mo, W), die aufgrund ihrer Reaktivität eine Reaktionsdurchführung bei moderaten Temperaturen erlauben. So verläuft die Reaktion von $\left(\mathrm{CH}_{3} \mathrm{CN}\right)_{3} \mathrm{Cr}(\mathrm{CO})_{3}$ mit einem halben Moläquivalent Methylendicyclopentadien zu grünem bcpmCr ${ }_{2}(\mathrm{CO})_{6} 1$ mit den besten Ausbeuten (72\%) in THF bei $65^{\circ} \mathrm{C}$ und einer Dauer von $17 \mathrm{~h}$ (Gl. 1). Durch Temperaturerhöhung bzw. durch Wechsel des Lösungsmittels (z.B. Toluol) tritt eine Minderung der Ausbeuten zugunsten eines grünen, nicht näher zu charakterisierenden Öls ein.

\section{$\left(\mathrm{CH}_{3} \mathrm{CN}\right)_{3} \mathrm{M}(\mathrm{CO})_{3}$}

$M-C, M o, w$
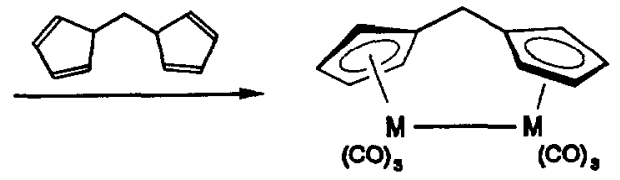

\begin{tabular}{c|c|c|c}
$M$ & $C_{1}$ & Mo & $W$ \\
\hline & 1 & 2 & 3
\end{tabular}

Zur Darstellung der roten Mo- und W-Komplexe 2 und 3 wird die Reaktionstemperatur auf $110^{\circ} \mathrm{C}$ erhöht, wobei nun Toluol als Lösungsmittel verwendet wird. Die Reaktionszeit wird auf $45 \mathrm{~h}$ gesteigert. Da sich unter diesen Bedingungen der eingesetzte Ligand bereits merklich zu zersetzen beginnt, wird von diesem ein dreifacher Überschuß verwendet. Die Zugabe erfolgt portionsweise innerhalb der ersten 22 h. Die Reaktion liefert die Produkte 2 und 3 in guten bis sehr guten Ausbeuten (78 bzw. 49\%) neben rot gefärbten "Ölen". * Obwohl vor allem die

\footnotetext{
* Anmerkung bei der Korrektur: Die Oxidation von $\left[\mathrm{bcpm} \mathrm{M} \mathrm{M}_{2}(\mathrm{CO})_{6}\right] \mathrm{Na}_{2}(\mathrm{M}=\mathrm{Mo}, \mathrm{W})$ mit $\mathrm{Fe}_{2}\left(\mathrm{SO}_{4}\right)_{3}$ gibt 2 und 3 in jeweils 28\% Ausbeute [T.E. Bitterwolf und A.L. Rheingold, Organometallics, 10 (1991) 3856].
} 
Bildung von 2 und 3 in einem Temperaturbereich erfolgt, in dem auch COEliminierung denkbar wäre [8], sind von carbonylärmeren Komplexen des Typs bcpmM $_{2}(\mathrm{CO})_{4}$ mit einer Metall-Metall-Dreifachbindung nicht einmal Spuren beobachtbar. Weitere photochemische und thermische Versuche, 1-3 zu decarbonylieren, blieben ebenfalls erfolglos.

In den Infrarotspektren (Tab. 1) von 1-3 weisen die Absorptionen der COStreckschwingung auf die niedrige Molekülsymmetrie dieser Verbindungen hin. Aufgenommen in $\mathrm{KBr}$ oder in Toluol ergeben sich für diese Liganden fünf bis sechs Absorptionsbanden, die in Bandenlage und -charakteristik denen der analogen, silylverbrückten Verbindungen $\mathrm{Me}_{2} \mathrm{Si}_{2}\left[\mathrm{C}_{5} \mathrm{H}_{4} \mathrm{M}(\mathrm{CO})_{3}\right]_{2} \quad(\mathrm{M}=\mathrm{Cr}, \mathrm{Mo}, W)$ ähneln [7]. Die $\mathrm{C}-\mathrm{H}-$ bzw. C-C-Valenz- und -Deformationsschwingungen des organischen Liganden in den Bereichen um 3050, 2900, 1400 und $850 \mathrm{~cm}^{-1}$ sind in allen Spektren nahezu identisch, liefern jedoch keine weitere strukturelle Information.

In den ${ }^{1}$ H-NMR-Spektren (Tab. 1) sind bei $297 \mathrm{~K}$ neben dem Singulett der Methylenprotonen für dic Ringprotonen des Biscyclopentadienylmethanliganden zwei (1) bzw. ein $(2,3)$ Resonanzsignale erkennbar. In den von 1 und 2 bei $193 \mathrm{~K}$ aufgenommenen Spektren ergeben die Ringprotonen dagegen je vier wohl separierte Signale.

Das für die bcpmM $\mathbf{B}_{2}(\mathrm{CO})_{6}$-Komplexe erhaltene spektroskopische Datenmaterial deutet auf einen von Heck an $\left.\mathrm{Me}_{2} \mathrm{Si}_{5} \mathrm{C}_{5} \mathrm{H}_{4} \mathrm{~W}(\mathrm{CO})_{3}\right]_{2}$ [7] bereits gut untersuchten Topomerisierungsproze $B$ mit näherungsweise ähnlichen Aktivierungsparametern hin. Demnach handelt es sich um die in Gl. 2 gezeigte, bei Raumtemperatur verlaufende Umwandlung der chiralen Verbindung in ihr Enantiomeres. Eine Homolyse der Metall-Metall-Bindung während der Topomerisierung ist auszuschließen.
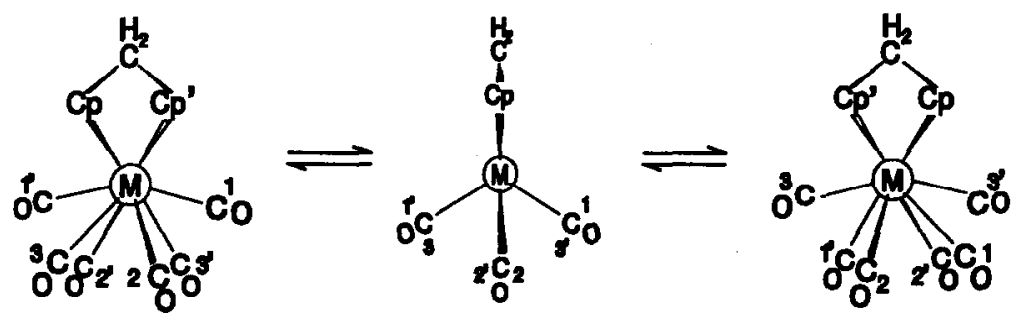

bcpmMo ${ }_{2}\left(\mathrm{CO}_{6} \mathrm{Cl}_{2}\right.$ (4)

Im Gegensatz zur Chemie der silylverbrückten Homologen, deren C-Si-Bindungen säureempfindlich sind [9], läßt sich zur Synthese von 4 die seit langem bewährte Methode zur Darstellung von $\mathrm{CpMo}(\mathrm{CO})_{3} \mathrm{Cl}$ anwenden [10]. So gelangt man durch Umsetzung von Dilithium-Biscyclopentadienylmethan mit $\mathrm{Mo}(\mathrm{CO})_{6}$ in nahezu siedendem THF zu extrem luftempfindlichem $\mathrm{Li}_{2}\left[\mathrm{bcpmMo}{ }_{2}(\mathrm{CO})_{6}\right]$, das sofort durch Ansäuern der Reaktionslösung in die entsprechende DihydridoVerbindung überführt wird. Durch darauffolgendes Versetzen dieser Lösung mit $\mathrm{CCl}_{4}$ gelangt man zu rotem bcpmMo $\mathrm{M}_{2}(\mathrm{CO})_{6} \mathrm{Cl}_{2} 4$ (Gl. 3), das chromatographisch gereinigt werden muß und schließlich in $43 \%$ Ausbeute anfällt. Ein oranges Nebenprodukt ist laut FD-MS ein einkerniger Komplex der Zusammensetzung $\mathrm{C}_{20} \mathrm{H}_{17} \mathrm{MoClO}_{3}$. Aufgrund der Empfindlichkeit und der geringen Menge dieses 
Tabelle 1

IR- und ${ }^{1} \mathrm{H}$-NMR-Daten der Komplexe 1-3

\begin{tabular}{|c|c|c|c|c|c|}
\hline & \multirow[t]{2}{*}{ IR, $v(\mathrm{CO})\left(\mathrm{cm}^{-1}\right)$} & \multirow[t]{2}{*}{ Solvens } & \multicolumn{2}{|l|}{${ }^{1} \mathrm{H}-\mathrm{NMR}$} & \multirow[t]{2}{*}{ Solvens $^{a}(T(\mathrm{~K}))$} \\
\hline & & & $\delta\left(\mathrm{CH}_{2}\right)$ & $\delta(\mathrm{Cp}-H)$ & \\
\hline \multirow[t]{2}{*}{1} & $\begin{array}{c}2004 \mathrm{vs}, 1950 \mathrm{vs}, 1910 \mathrm{vs}, \\
1898 \mathrm{vs}, 1885 \mathrm{vs}, 1880 \mathrm{~s}\end{array}$ & $\mathbf{K B r}$ & $3.38(\mathrm{~s}, 2 \mathrm{H})$ & $4.89(\mathrm{~s}, 4 \mathrm{H}), 4.96(4 \mathrm{H})$ & $\mathrm{CDCl}_{3}(297)$ \\
\hline & $\begin{array}{l}2004 \mathrm{vs}, 1954 \mathrm{vs}, 1930 \mathrm{vs}, \\
1912 \mathrm{vs}, 1895 \mathrm{vs}, 1863 \mathrm{sh}\end{array}$ & Toluol & $\begin{array}{l}2.53(\mathrm{~s}, 2 \mathrm{H}) \\
2.16(\mathrm{~s}, 2 \mathrm{H})\end{array}$ & $\begin{array}{l}4.13(\mathrm{~s}, 4 \mathrm{H}), 4.26(4 \mathrm{H}) \\
3.23(\mathrm{~m}, 2 \mathrm{H}), 3.74(\mathrm{~m}, 2 \mathrm{H}), \\
3.99(\mathrm{~m}, 2 \mathrm{H}), 4.78(\mathrm{~m}, 2 \mathrm{H})\end{array}$ & $\begin{array}{l}\text { Toluol- } d_{8}(297) \\
\text { Toluol- } d_{8}(193)\end{array}$ \\
\hline \multirow[t]{2}{*}{2} & $\begin{array}{l}\text { 2005vs, 1959vs, } 1910 \mathrm{vs,} \\
\text { 1897vs, } 1877 \mathrm{~s}\end{array}$ & $\mathbf{K B r}$ & $3.56(\mathrm{~s}, 2 \mathrm{H})$ & $5.29(8 \mathrm{H})^{b}$ & $\mathrm{CDCl}_{3}(297)$ \\
\hline & $\begin{array}{l}2005 v s, 1967 \mathrm{vs,} 1924 \mathrm{vs}, \\
1908 \mathrm{vs}, 1888 \mathrm{vs}, 1856 \mathrm{sh}\end{array}$ & Toluol & $\begin{array}{l}2.64(\mathrm{~s}, 2 \mathrm{H}) \\
2.24(\mathrm{~s}, 2 \mathrm{H})\end{array}$ & $\begin{array}{l}4.51(8 \mathrm{H})^{b} \\
3.62(\mathrm{~m}, 2 \mathrm{H}), 4.08(\mathrm{~m}, 2 \mathrm{H}) \\
4.36(\mathrm{~m}, 2 \mathrm{H}), 4.93(\mathrm{~m}, 2 \mathrm{H})\end{array}$ & $\begin{array}{l}\text { Toluol- } d_{8}(297) \\
\text { Toluol- } d_{8}(193)\end{array}$ \\
\hline \multirow[t]{2}{*}{3} & $\begin{array}{l}2000 \mathrm{vs}, 1952 \mathrm{vs}, 1896 \mathrm{vs} \\
1885 \mathrm{vs}, 1865 \mathrm{vs}\end{array}$ & $\mathrm{KBr}$ & $3.90(\mathrm{~s}, 2 \mathrm{H})$ & $5.33(8 \mathrm{H})$ & $\mathrm{CDCl}_{3}(297)$ \\
\hline & $\begin{array}{l}\text { 2005vs, 1964vs, 1925vs, } \\
\text { 1900sh, 1880sh }\end{array}$ & Toluol & & & \\
\hline
\end{tabular}

${ }^{a}$ Gerät Bruker WM 250; i-TMS. ${ }^{b}$ Breites Singulett.

orangen Nebenproduktes war keine weitere Charakterisierung möglich.

$$
\begin{aligned}
2 \mathrm{Mo}(\mathrm{CO})_{6}+\mathrm{Li}_{2}[\mathrm{bcpm}] \stackrel{-6 \mathrm{CO}}{\longrightarrow} \mathrm{Li}_{2}[\text { bcpmMo } & \left.(\mathrm{CO})_{6}\right] \\
& \downarrow+\mathrm{H}^{+} \\
& \text {bcpmMo }_{2}(\mathrm{CO})_{6} \mathrm{H}_{2} \stackrel{\mathrm{CCl}_{4}}{\longrightarrow} \text { bcpmMo }_{2}(\mathrm{CO})_{6} \mathrm{Cl}_{2}
\end{aligned}
$$

4

Die Zusammensetzung von bcpmMo $\mathrm{M}_{2}(\mathrm{CO})_{6} \mathrm{Cl}_{2}$ (4) wurde massenspektrometrisch und elementaranalytisch abgesichert. Das Infrarotspektrum von 4 zeigt die zwei erwarteten Absorptionsbanden der Streckschwingung terminaler Carbonylliganden bei 2050 und $1965 \mathrm{~cm}^{-1}$. Dem ${ }^{1} \mathrm{H}-\mathrm{NMR}$-Spektrum $\left(\mathrm{CDCl}_{3} / \mathrm{i}-\mathrm{TMS}\right)$ sind für die Methylenprotonen des Biscyclopentadienylmethanliganden ein Singulett bei 3.40 ppm und für die Ringprotonen zwei pseudo-Tripletts bei 5.25 bzw. $5.77 \mathrm{ppm}$ zu entnehmen.

Röntgenstrukturanalyse von bcpmCr $\mathrm{Cr}_{2}(\mathrm{CO})_{6}(1)$

Der chirale Aufbau von $1\left(C_{2}\right.$-Symmetrie) wurde durch eine an einem aus Toluol bei $-18^{\circ} \mathrm{C}$ gezogenen Einkristall durchgeführte Röntgenstrukturanalyse (Tab. 2, 3) gesichert. Seine helikale Chiralität erhält 1 durch die Verwindung des Biscyclopentadienylmethan-Liganden. Der Torsionswinkel $\mathrm{Cp}(1)_{\text {Mitte }}-\mathrm{Cr}(1) /$ $\mathrm{Cr}(2)-\mathrm{Cp}(2)$ Mitte beträgt $44.7^{\circ}$. Der Winkel am verbrückenden Methylenkohlenstoff ist gegenüber einem idealen Tetraederwinkel um $5.4^{\circ}$ aufgeweitet. Die $\mathrm{Cr}(\mathrm{CO})_{3}$ Baugruppen befinden sich zueinander in einer sterisch günstigen gauche-Anordnung, wobei die CO-Gruppen 2 und 3 fast ekliptisch zu den Gruppen 5 und 7 stehen. Damit ist 1 in seinem Aufbau analog zu $\mathrm{Me}_{2} \mathrm{Si}_{5}\left[\mathrm{C}_{5} \mathrm{H}_{4} \mathrm{~W}(\mathrm{CO})_{3}\right]_{2}$ [7]. Der 
Tabelle 2

Atomkoordinaten $\left(\times 10^{4}\right)$ und thermische Parameter $\left(U_{\text {eq }} \times 10^{3}\right)$ von $\mathrm{bcpmCr}_{2}(\mathrm{CO})_{6}(1)$

\begin{tabular}{lrrrr}
\hline Atom & \multicolumn{1}{c}{$\boldsymbol{y}$} & \multicolumn{1}{l}{$\boldsymbol{l}$} & $U_{\text {eq }}$ \\
\hline Cr(1) & $41(1)$ & $3302(1)$ & $1218(1)$ & $34(1)$ \\
Cr(2) & $1030(1)$ & $1455(1)$ & $1580(1)$ & $34(1)$ \\
$C(2)$ & $566(5)$ & $4386(4)$ & $1156(4)$ & $69(2)$ \\
O(2) & $931(4)$ & $5065(3)$ & $1132(4)$ & $110(3)$ \\
$C(3)$ & $604(4)$ & $3403(3)$ & $2306(3)$ & $44(2)$ \\
O(3) & $932(3)$ & $3560(3)$ & $2969(3)$ & $72(2)$ \\
$C(4)$ & $1012(4)$ & $3160(3)$ & $376(3)$ & $46(2)$ \\
O(4) & $1554(3)$ & $3150(3)$ & $-194(3)$ & $73(2)$ \\
$C(5)$ & $1851(4)$ & $1009(3)$ & $2408(3)$ & $49(2)$ \\
$O(5)$ & $2378(3)$ & $715(3)$ & $2916(3)$ & $76(2)$ \\
$C(6)$ & $273(4)$ & $1694(3)$ & $2547(3)$ & $48(2)$ \\
$O(6)$ & $-176(3)$ & $1775(3)$ & $3165(3)$ & $72(2)$ \\
$C(7)$ & $2087(3)$ & $2240(3)$ & $1521(3)$ & $40(2)$ \\
$O(7)$ & $2789(3)$ & $2677(3)$ & $1506(2)$ & $56(1)$ \\
$C(1)$ & $-787(4)$ & $1714(3)$ & $51(3)$ & $53(2)$ \\
$C(11)$ & $-1114(4)$ & $3352(3)$ & $218(3)$ & $46(2)$ \\
$C(12)$ & $-1070(3)$ & $2515(3)$ & $554(3)$ & $39(1)$ \\
$C(13)$ & $-1350(3)$ & $2562(3)$ & $1428(3)$ & $44(2)$ \\
$C(14)$ & $-1554(4)$ & $3422(3)$ & $1628(3)$ & $49(2)$ \\
$C(15)$ & $-1406(4)$ & $3905(3)$ & $882(3)$ & $47(2)$ \\
$C(16)$ & $764(4)$ & $110(3)$ & $1248(4)$ & $56(2)$ \\
$C(17)$ & $1490(4)$ & $458(3)$ & $693(3)$ & $52(2)$ \\
$C(18)$ & $1030(4)$ & $1105(3)$ & $211(3)$ & $46(2)$ \\
$C(19)$ & $12(4)$ & $1164(3)$ & $464(3)$ & $41(1)$ \\
$C(20)$ & $-146(4)$ & $563(3)$ & $1113(3)$ & $50(2)$ \\
\hline & & & & \\
\hline
\end{tabular}

Tabelle 3

Ausgewählte Bindungslängen ( $\AA$ ) und -winkel $\left(^{\circ}\right)$ von bcpmCr ${ }_{2}(\mathrm{CO})_{6}(1)$

\begin{tabular}{|c|c|c|c|}
\hline $\mathrm{Cr}(1)-\mathrm{Cr}(2)$ & $3.211(2)$ & & \\
\hline $\operatorname{Cr}(1)-C(2)$ & $1.829(6)$ & $\mathrm{Cr}(2)-\mathrm{C}(5)$ & $1.830(5)$ \\
\hline $\mathrm{Cr}(1)-\mathrm{C}(3)$ & $1.865(5)$ & $\mathrm{Cr}(2)-\mathrm{C}(6)$ & $1.854(5)$ \\
\hline $\mathrm{Cr}(1)-\mathrm{C}(4)$ & $1.858(5)$ & $\mathrm{Cr}(2)-\mathrm{C}(7)$ & $1.866(5)$ \\
\hline $\mathrm{Cr}(1)-\mathrm{C}(11)$ & $2.193(5)$ & $\mathrm{Cr}(2)-\mathrm{C}(16)$ & $2.185(5)$ \\
\hline $\mathrm{Cr}(1)-\mathrm{C}(12)$ & $2.182(5)$ & $\mathrm{Cr}(2)-\mathrm{C}(17)$ & $2.168(5)$ \\
\hline $\mathrm{Cr}(1)-\mathrm{C}(13)$ & $2.204(5)$ & $\mathrm{Cr}(2)-\mathrm{C}(18)$ & $2.209(5)$ \\
\hline $\mathrm{Cr}(1)-\mathrm{C}(14)$ & $2.226(5)$ & $\operatorname{Cr}(2)-C(19)$ & $2.255(5$ \\
\hline $\mathrm{Cr}(1)-\mathrm{C}(15)$ & $2.206(5)$ & $\mathrm{Cr}(2)-\mathrm{C}(20)$ & $2.215(5)$ \\
\hline$C(1)-C(12)$ & $1.521(7)$ & $C(1)-C(19)$ & $1.510(7)$ \\
\hline$C(2)-C r(1)-C(3)$ & $79.5(3)$ & $\mathrm{C}(5)-\mathrm{Cr}(2)-\mathrm{C}(6)$ & $79.9(2)$ \\
\hline$C(2)-C r(1)-C(4)$ & $78.8(3)$ & $C(5)-C r(2)-C(7)$ & $80.4(2)$ \\
\hline$C(3)-C r(1)-C(4)$ & $112.1(2)$ & $C(6)-\operatorname{Cr}(2)-C(7)$ & $108.6(2)$ \\
\hline $\mathrm{Cr}(2)-\mathrm{Cr}(1)-\mathrm{C}(2)$ & $132.7(2)$ & $\mathrm{Cr}(1)-\mathrm{Cr}(2)-\mathrm{C}(5)$ & $135.1(2)$ \\
\hline $\operatorname{Cr}(2)-\operatorname{Cr}(1)-C(3)$ & $75.5(2)$ & $\mathrm{Cr}(1)-\mathrm{Cr}(2)-\mathrm{C}(6)$ & $75.0(2)$ \\
\hline $\mathrm{Cr}(2)-\mathrm{Cr}(1)-\mathrm{C}(4)$ & $74.5(2)$ & $C_{r}(1)-C_{r}(2)-C(7)$ & $73.4(2)$ \\
\hline $\mathrm{Cr}(2)-\mathrm{Cr}(1)-\mathrm{Cp}(1)_{\text {Mitte }}$ & 108.9 & $\mathrm{Cr}(1)-\mathrm{Cr}(2)-\mathrm{Cp}(2)_{\text {Mitte }}$ & 109.9 \\
\hline$C(12)-C(1)-C(19)$ & $114.7(4)$ & & \\
\hline
\end{tabular}




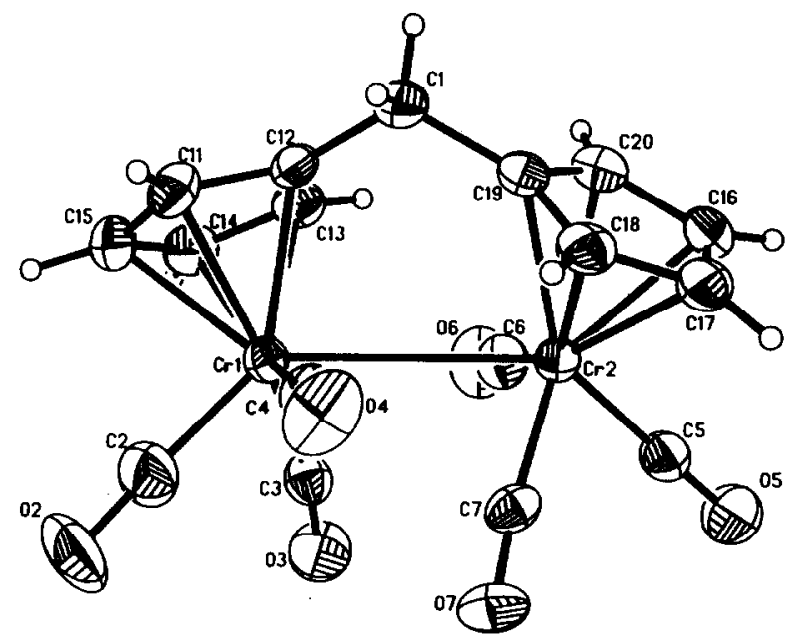

Fig. 1. Molekülstruktur von bcpmCr ${ }_{2}(\mathrm{CO})_{6}, 1$ (oRTEP-Zeichnung).

Abstand der Chromzentren in 1 ist mit 3.211(2) $\AA$ sogar etwas größer als $d_{\mathrm{W}-\mathrm{W}}$ in $\mathrm{Me}_{2} \mathrm{Si}_{5}\left[\mathrm{C}_{5} \mathrm{H}_{4} \mathrm{~W}(\mathrm{CO})_{3}\right]_{2}$ (3.196(1) $\AA$ ), er ist jedoch trotz der "Ligandenzange" nur geringfügig kürzer ( $\mathrm{ca}$. $0.12 \AA$ ) als in den unverbrückten Komplexen des Typs $\mathrm{Cp}_{2} \mathrm{Cr}_{2}(\mathrm{CO})_{6}$ [11]. Aus diesem Grund ist wohl die repulsive Wirkung der Carbonylliganden als die den Metall-Metall-Abstand bestimmende Größe anzusehen.

\section{Experimenteller Teil}

Sämtliche Arbeiten wurden unter Schutzgas $\left(\mathrm{N}_{2}\right)$ und unter Verwendung von trockenen, $\mathrm{N}_{2}$-gesättigten Lösungsmitteln durchgeführt. Die FD-Massenspektren wurden an einem MAT 311A-Spektrometer aus Toluol-Lösungen aufgenommen. Methylendicyclopentadien [12] und die $\left(\mathrm{CH}_{3} \mathrm{CN}\right)_{3} \mathrm{M}(\mathrm{CO})_{3}-\mathrm{Komplexe}$ [13] wurden nach bereits beschriebenen Verfahren erhalten.

\section{Darstellung von bcpmCr${ }_{2}(\mathrm{CO})_{6}(1)$}

Eine rotbraune Lösung von $4.14 \mathrm{~g}(16.00 \mathrm{mmol})\left(\mathrm{CH}_{3} \mathrm{CN}\right)_{3} \mathrm{Cr}(\mathrm{CO})_{3}$ und $1.44 \mathrm{~g}$ (10.00 mmol) bcpmH be $_{2}$ in $100 \mathrm{ml}$ THF wird $17 \mathrm{~h}$ bei $65^{\circ} \mathrm{C}$ gerührt. Die nunmehr dunkelgrüne Reaktionslösung wird vom Lösungsmittel befreit, das ölige Rohprodukt in einem Toluol/Pentan-Gemisch (2/1) aufgenommen und an $\mathrm{SiO}_{2}$ (Akt. II-III, Säule $\left.3 \times 20 \mathrm{~cm}, 10^{\circ} \mathrm{C}\right)$ chromatographiert. Mit Toluol/Pentan $(3 / 2)$ eluiert man zunächst eine grüne ölige Zone, die organische Nebenprodukte enthält. Die darauf folgende grüne Hauptzone enthält 1 in $69 \%$ Ausbeute. 1 wird aus Toluol bei $-18^{\circ} \mathrm{C}$ umkristallisiert.

Elementaranalyse: 1: Gef.: C, 49.08; $\mathrm{H}, 2.34 . \mathrm{C}_{17} \mathrm{H}_{10} \mathrm{Cr}_{2} \mathrm{O}_{6}$ (414.25) ber.: $\mathrm{C}$, 49.29; H, 2.43\%. Molmasse 413.80 (Schwerpunkt; FD-MS).

Darstellung von bcpmM $\mathrm{M}_{2}(\mathrm{CO})_{6}(\mathrm{M}=\mathrm{Mo}, \mathrm{W})(2,3)$

$25 \mathrm{mmol}\left(\mathrm{CH}_{3} \mathrm{CN}\right)_{3} \mathrm{M}(\mathrm{CO})_{3}(\mathrm{M}=\mathrm{Mo}, \mathrm{W})$ werden in ca. $120 \mathrm{ml}$ Toluol gelöst. Bei $110^{\circ} \mathrm{C}$ werden insgesamt $38 \mathrm{mmol} \mathrm{bcpmH}_{2}$ in $1.0 \mathrm{~g}$ Portionen innerhalb von 22 
h zugegeben. Man rührt danach weitere $23 \mathrm{~h}$ bei gleicher Temperatur. Nach Abziehen des Lösungsmittels werden die in beiden Fällen roten, öligen Rohprodukte an $\mathrm{SiO}_{2}$ (wassergekühlte Säule $2 \times 30 \mathrm{~cm}$ ) mit Toluol/Pentan $(3 / 2)$ als Eluens chromatographiert. Die als erste rote Zone laufenden organischen Beiprodukte werden verworfen, die jeweils zweiten roten Zonen enthalten 2 bzw. 3 .

Elementaranalyse: 2: Gef.: $\mathrm{C}, 41.00 ; \mathrm{H}, 2.05$. $\mathrm{C}_{17} \mathrm{H}_{10} \mathrm{Mo}_{2} \mathrm{O}_{6}(501.96)$ ber.: $\mathrm{C}$, 40.68; H, 2.01\%. Molmasse 502.00 (Schwerpunkt; FD-MS); 3: Gef.: C, 30.00; H, 1.49. $\mathrm{C}_{17} \mathrm{H}_{10} \mathrm{~W}_{2} \mathrm{O}_{6}$ (677.96) ber.: $\mathrm{C}, 30.12 ; \mathrm{H}, 1.49 \%$. Molmasse 678.40 (Schwerpunkt; FD-MS).

Darstellung von bcpmMo ${ }_{2}(\mathrm{CO})_{6} \mathrm{Cl}_{2}$ (4)

$3.50 \mathrm{~g}\left(23 \mathrm{mmol}\right.$ ) frisch dargestelltes bcpmLi ${ }_{2}$ (aus bcpmH ${ }_{2}$ und ${ }^{\mathrm{n}} \mathrm{BuLi}$ in Pentan, $\left.0^{\circ} \mathrm{C}\right)$ werden in $100 \mathrm{ml}$ THF suspendiert. Nach Zugabe von $3.00 \mathrm{~g}(12.50$ mmol) $\mathrm{Mo}(\mathrm{CO})_{6}$ rührt man $6 \mathrm{~h}$ unter Lichtausschluß bei $75^{\circ} \mathrm{C}$ Ölbadtemperatur. Nach Abziehen des Lösungsmittels wird der grüngelbe, ölige Rückstand in $\mathrm{ca} .100$ $\mathrm{ml} \mathrm{H}_{2} \mathrm{O}$ aufgenommen und mit $30 \mathrm{ml}$ Eisessig unter Eiskühlung versetzt. Nach 10 min Rühren werden $20 \mathrm{ml} \mathrm{CCl}_{4}$ und $100 \mathrm{ml}$ Ether zugesetzt und noch $30 \mathrm{~min}$ gerührt. Die dann intensiv rote organische Phase wird abgetrennt und die verbleibende wäßrige Phase mit insgesamt $150 \mathrm{ml}$ Ether ausgeschüttelt. Die vereinigten organischen Phasen werden vom Lösungsmittel befreit und in $50 \mathrm{ml}$ Aceton erneut gelöst. Diese Lösung wird mit $\mathrm{Na}_{2} \mathrm{SO}_{4}$ und Aktivkohle versetzt, 30 min gerührt, danach filtriert und vom Lösungsmittel befreit. Bei der Chromatographie des verbliebenen rotorangen Rückstandes $\left(\mathrm{SiO}_{2} /\right.$ Toluol; Säule $\left.3 \times 20 \mathrm{~cm}\right)$ entwickelt sich zunächst eine gelbe Zone, die laut FD-Massenspektrum eine einkernige Verbindung der Zusammensetzung $\mathrm{C}_{20} \mathrm{H}_{17} \mathrm{MoClO}_{3}(\mathrm{~m} / e=432)$ enthält. Eine zweite, rote Zone liefert bcpmMo ${ }_{2}(\mathrm{CO})_{6} \mathrm{Cl}_{2}(4)$ in $43 \%$ Ausbeute.

Elementaranalyse: 4: Gef.: $\mathrm{C}, 35.86 ; \mathrm{H}, 1.95 . \mathrm{C}_{17} \mathrm{H}_{10} \mathrm{Cl}_{2} \mathrm{Mo}_{2} \mathrm{O}_{6}(572.86)$ ber.: $\mathrm{C}$, 35.64; H, 1.76\%. Molmasse 573.00 (Schwerpunkt; FD-MS).

Röntgenographische Daten von Komplex bcpmCr ${ }_{2}(\mathrm{CO})_{6}(1)$

Schwarzer Kristall $\left(0.17 \times 0.63 \times 0.68 \mathrm{~mm}^{3}\right)$, rhombisch $D_{2 \mathrm{~h} / 15}, P b c a,(61)$; Zellkonstanten: $a$ 13.13(1), $b$ 15.56(1), $c$ 15.63(1) $\AA$; $V 3237.03 \AA^{3}, Z=8$; empirische Absorptionskorrektur ( $\psi$-scan Messung: 8 Reflexe $5.0<2 \theta<41.0^{\circ}$. Transmiss. Faktor (min./max.) 0.788/0.959), $\mu 1.34 \mathrm{~mm}^{-1} . F(000) 1664, d$ (röntg) $=1.70$ $\mathrm{g} / \mathrm{cm}^{3}$; Syntex R3. Mo- $K_{\alpha}$-Strahlung, Graphit-Monochromator, im vermessenen Bereich 4697 mögliche Reflexe; unabhängige Reflexe $3056(I>2.5 \sigma(I))$. Die Struktur wurde gelöst mittels Patterson-, Fourier- und Differenzfouriersynthesen; die H-Atome wurden mit Hilfe des SHELXTL-Unterprogramms HFIX [14] fixiert; $R=0.054, \quad R_{\mathrm{w}}=0.049 ;$ Restelektronendichte (max. $/$ min.) $0.43 /-0.87 \mathrm{e} / \AA^{3}$, shift/esd (mean/max.) $-/ 0.004$, GOOF $=3.26$.

\section{Literatur}

1 T.B. Bitterwolf, J. Organomet. Chem., 312 (1986) 197.

2 S.A.R. Knox, K.A. Macpherson, A.G. Orpen und M.C. Rendle, J. Chem. Soc., Dalton Trans., (1989) 1807.

3 H.E. Bryndzay und R.G. Bergman, J. Am. Chem. Soc., 101 (1979) 4766.

4 H. Werner, H.-J. Scholz und R. Zolk, Chem. Ber., 118 (1985) 4531. 
5 T.B. Bitterwolf, A. Gambaro, F. Gottardi und G. Valle, Organometallics, 10 (1991) 1416.

6 H.-J. Scholz und H. Werner, J. Organomet. Chem., 303 (1986) C8.

7 W. Abriel und J. Heck, J. Organomet. Chem., 302 (1986) 363; J. Heck, K.A. Kriebisch und H. Mellinghoff, Chem. Ber., 121 (1981) 1753.

8 M.D. Curtis und R.J. Klingler, J. Organomet. Chem., 161 (1978) 23; M.D. Curtis und M.S. Hay, Inorg. Synth., 28 (1991) 152.

9 R. Baumann und W. Malisch, J. Organomet. Chem., 303 (1986) C33.

10 T.S. Piper und G. Wilkinson, Inorg. Nucl. Chem., 3 (1956) 104.

11 L.-Y.-Goh, M.J. D'Aniello, S. Slater, E.L. Muetterties, I. Tavanaiepour, M.I. Chang, M.F. Fredrich und V.W. Day, Inorg. Chem., 18 (1979) 192; T.J. Jaeger und M.C. Baird, Organometallics, 7 (1988) 2074; L.-Y. Goh, T.W. Hambley, D.J. Darensbourg und J. Reibenspies, J. Organomet. Chem., 381 (1990) 349.

12 H. Schaltegger, M. Neuenschwander und D. Meuche, Helv. Chim. Acta, 48 (1965) 955; N.E. Schore, C.S. Ilenda, M.A. White, H.E. Bryndzay, M.G. Matturro und R.G. Bergman, J. Am. Chem. Soc., 106 (1984) 7451.

13 G.J. Kubas und L.S. van der Sluys, Inorg. Synth., 28 (1991) 29.

14 G.M. Sheldrick; sHELXTL-Programm (Unterprogramm HFIX), Universität Göttingen, Deutschland, 1983. 